\title{
Testing for Structural Change in Conditional Models
}

\author{
Bruce E. Hansen* \\ Boston College ${ }^{\dagger}$ \\ January 1996 \\ Revised: May 1998
}

\begin{abstract}
In the past decade, we have seen the development of a new set of tests for structural change of unknown timing in regression models, most notably the SupF statistic of Andrews (1993), the ExpF and AveF statistics of Andrews-Ploberger (1994), and the L statistic of Nyblom (1989). The distribution theory used for these tests is primarily asymptotic, and has been derived under the maintained assumption that the regressors are stationary. This excludes structural change in the marginal distribution of the regressors. As a result, these tests technically cannot discriminate between structural change in the conditional and marginal distributions. This paper attempts to remedy this deficiency by deriving the large sample distributions of the test statistics allowing for structural change in the marginal distribution of the regressors. We find that the asymptotic distributions of the SupF, ExpF, AveF and L statistics are not invariant to structural change in the regressors. To solve the size problem, we introduce a "fixed regressor bootstrap" which achieves the first-order asymptotic distribution, and appears to possess reasonable size properties in small samples. Our bootstrap theory allows for arbitrary structural change in the regressors, including structural shifts, polynomial trends, and exogenous stochastic trends. It allows for lagged dependent variables and heteroskedastic error processes.
\end{abstract}

*This research was supported by a grant from the National Science Foundation and a Alfred P. Sloan Foundation Research Fellowship. Thanks go to Byeongseon Seo for excellent research assistance, to James Stock and Mark Watson for useful conversations, and to four referees for helpful comments.

${ }^{\dagger}$ Department of Economics, Boston College, Chestnut Hill MA 02167-3806. bruce.hansen@bc.edu. Homepage: http://www2.bc.edu/ ${ }^{2}$ hansenb/ 


\section{Introduction}

There has been a recent surge of interest in tests for constancy of parameters in dynamic econometric models. The classic approach of assuming that the date of structural change is known has been replaced by testing procedures which do not presuppose such knowledge. Particularly important contributions include Nyblom's (1989) test for martingale parameter variation, Andrews' (1993) asymptotic theory for Quandt's (1960) test for a one-time parameter shift, and the exponentially-weighted tests of Andrews and Ploberger (1994). There appears to be considerable interest in the practical implications of these tests for econometric practice; see the recent exploratory work of Stock and Watson (1996).

The distribution theory referenced above has been derived under the assumption that the conditioning variables are stationary. This might not be a desirable assumption in practice. Consider the standard linear regression model

$$
y_{n i}=x_{n i}^{\prime} \beta_{n i}+e_{n i}, \quad i=1, \ldots, n,
$$

with $\sigma^{2}=E\left(e_{n i}^{2}\right)<\infty$. When we test for structural change in equation (1), we are typically interested in whether or not $\beta_{n i}$ is constant, and not particularly concerned with the distribution of $x_{n i}$. Thus the question of structural change in (1) is conceptually distinct from the question of whether or not $x_{n i}$ is stationary. Note that we have written the variables in (1) using array notation. This will facilitate large sample distribution assumptions allowing for non-stationarity in these processes, but otherwise has no important content.

In fact, it is often of particular interest to test for structural change in the conditional relationship (1) when it is known that the distribution of the conditioning variable $x_{n i}$ has experienced a structural change. Indeed, constancy of (1) in the presence of a shift in the marginal distribution of $x_{n i}$ is part of the definition of super exogeneity proposed by Engle, Hendry and Richard (1983). Tests of this hypothesis have been discussed by Hendry (1988) and Engle and Hendry (1993).

If the distributions of tests for constancy of $\beta_{n i}$ are robust to structural change in $x_{n i}$, then we would have nothing to worry about, as a significant test statistic could be unambiguously 
interpreted as evidence for structural instability in $\beta_{n i}$. But this may not be the case. If the null distribution is affected by a structural change in $x_{n i}$, then a significant test statistic could indicate that there is instability in either $\beta_{n i}$ or $x_{n i}$ (a conclusion which is not of much interest), and if the distribution under the alternative is adversely affected, power could suffer.

In this paper we carefully explore the asymptotic distributions allowing for structural change in the regressors. In section 2 we describe the model and test statistics, allowing for a one-time structural change in the regression parameters. In section 3 we describe the firstorder asymptotic theory. We consider both asymptotically stationary and non-stationary processes, where the latter includes structural change in the regressors. We find that the distributions are different for non-stationary regressors. To quantify these differences, section 4 explores the important special case of a single structural change in the marginal process. We find that the size and power distortions can be quite large. In particular, Nyblom's L statistic is asymptotically conservative, and the size distortion of Quandt's SupF statistic is potentially unbounded. In section 5 we discuss asymptotically valid inference based on a bootstrap distribution. In a simulation experiment, we find that this bootstrap technique works quite well when compared with the conventional asymptotic tests. Section 6 concludes. Proofs are presented in an Appendix.

Throughout the paper, $I(\cdot)$ denotes the indicator function, $[\cdot]$ denotes integer part, and " $\Rightarrow$ " denotes weak convergence with respect to the uniform metric over $r \in[0,1]$.

\section{$2 \quad$ Model and Tests}

The conditional distribution of $y_{n i}$ given $x_{n i}$ takes the form of a linear regression (1) where $y_{n i}$ is real-valued and $x_{n i}$ is an $m$-vector, and structural change in the conditional distribution arises through the coefficient $\beta_{n i}$. The structural change in $\beta_{n i}$ takes the form

$$
\beta_{n i}=\left\{\begin{array}{cc}
\beta, & i<t_{0} \\
\beta+\theta_{n}, & i \geq t_{0} .
\end{array}\right.
$$

The parameter $t_{0} \in\left[t_{1}, t_{2}\right]$ indexes the relative timing of the structural shift, and $\theta_{n}$ indexes the magnitude of the shift. 
We are interested in tests of $H_{0}: \theta_{n}=0$ against $H_{1}: \theta_{n} \neq 0$. To examine asymptotic local power we will specify $H_{1}$ as local to $H_{0}$. Specifically, we assume that $\theta_{n}$ takes the form

$$
\theta_{n}=\delta \sigma / \sqrt{n}
$$

with $\delta$ fixed as $n \rightarrow \infty$. The parameter $\delta$ indexes the degree of structural change under the local alternative $H_{1}$. For concreteness, we collect our maintained assumptions:

Assumption 1 The linear regression model is (1)-(2)-(3). The error $e_{n i}$ is a martingale difference: $E\left(e_{n i} \mid \Im_{n i-1}\right)=0$ where $\Im_{n i-1}$ is the sigma-field generated by current $x_{n i}$ and lagged values of $\left(x_{n i}, e_{n i}\right)$. The sequence $e_{n i}^{2}$ satisfies a WLLN, so that $n^{-1} \sum_{i=1}^{n} e_{n i}^{2} \rightarrow p \sigma^{2}$. The parameters $\tau_{0}=t_{0} / n, \pi_{1}=t_{1} / n>0$ and $\pi_{2}=t_{2} / n<1$ are fixed as $n \rightarrow \infty$.

Sometimes we will add the assumption that $e_{n i}$ is conditionally homoskedastic:

$$
E\left(e_{n i}^{2} \mid \Im_{n i-1}\right)=\sigma^{2}, \quad \text { a.s. }
$$

Under $H_{0}$, model (1)-(2) reduces to $y_{n i}=x_{n i}^{\prime} \beta+e_{n i}$ which does not depend on $t_{0}$. Denote the ordinary least squares (OLS) estimator $\hat{\beta}$, residuals $\hat{e}_{i}$, and variance estimate $\hat{\sigma}^{2}=(n-m)^{-1} \sum_{i=1}^{n} \hat{e}_{i}^{2}$. Under the alternative $H_{1}: \theta_{n} \neq 0$, the model can be written as

$$
y_{n i}=x_{n i}^{\prime} \beta+x_{n i}^{\prime} \theta_{n} I\left(i \geq t_{0}\right)+e_{n i}
$$

For any fixed $t,(5)$ can be estimated by OLS, yielding estimates $\left(\hat{\beta}_{t}, \hat{\theta}_{t}\right)$, residuals $\hat{e}_{i t}$ and variance estimate $\hat{\sigma}_{t}^{2}=(n-2 m)^{-1} \sum_{i=1}^{n} \hat{e}_{i t}^{2}$. Let $\hat{t}=\operatorname{argmin} \hat{\sigma}_{t}^{2}$ denote the least squares estimate of the breakdate and set $\tilde{\beta}=\hat{\beta}_{\hat{t}}$ and $\tilde{e}_{i}=\hat{e}_{i \hat{t}}$.

The standard test for $H_{0}$ against $H_{1}$ for known $t$ (e.g., Chow (1960)) is the Wald statistic:

$$
F_{t}=\frac{(n-m) \hat{\sigma}^{2}-(n-2 m) \hat{\sigma}_{t}^{2}}{\hat{\sigma}_{t}^{2}} .
$$

The Wald statistic $F_{t}$ is equivalent to the likelihood ratio statistic when $e_{n i}$ is iid $N\left(0, \sigma^{2}\right)$.

We are interested in tests of $H_{0}$ when the true changepoint $t_{0}$ is unknown. Quandt (1960) proposed the likelihood ratio test which is equivalent to $\operatorname{SupF}_{n}=\sup _{t} F_{t}$, where the supremum is taken over $t \in\left(t_{1}, t_{2}\right)$. Andrews and Ploberger (1994) developed a theory of optimal testing, and suggested a related family of tests, including an exponentially 
weighted Wald test (optimal against distant alternatives) $\operatorname{ExpF}_{n}=\ln \int \exp \left(\mathrm{F}_{t} / 2\right) d w(t)$ and the average $\mathrm{F}$ test (optimal against very local alternatives) $\mathrm{AveF}_{n}=\int_{t} \mathrm{~F}_{t} d w(t)$, where $w$ is a measure putting weight $1 /\left(t_{2}-t_{1}\right)$ on each integer $t$ in the interval $\left[t_{1}, t_{2}\right]$. The Quandt and Andrews-Ploberger statistics assume that $\theta_{n}$ and $t_{0}$ are unknown parameters. Nyblom (1989) instead considered random structural change. Let $\theta_{n}$ and $t_{0}$ be random variables such that $E\left[\theta_{n}\left\{i=t_{0}\right\}\right]=0$ and $E\left[\theta_{n} \theta_{n}^{\prime}\left\{i=t_{0}\right\}\right]=\left(\sum_{t=1}^{n} x_{n i} x_{n i}^{\prime}\right)^{-1} \phi^{2}$ for some real number $\phi$. Nyblom's (1989) Lagrange Multiplier (LM) test for $\phi^{2}=0$ against $\phi^{2}>0$ rejects for large values of

$$
\mathrm{L}_{n}=\frac{1}{n \hat{\sigma}^{2}} \sum_{i=1}^{n}\left(\sum_{t=1}^{i} x_{n t} \hat{e}_{t}\right)^{\prime}\left(\sum_{t=1}^{n} x_{n t} x_{n t}^{\prime}\right)^{-1}\left(\sum_{t=1}^{i} x_{n t} \hat{e}_{t}\right)
$$

\section{Asymptotic Theory}

\subsection{Asymptotically Stationary Process}

Andrews (1993), Andrews-Ploberger (1994) and Nyblom (1989) assumed that the data are stationary. We now show that their distribution theory holds somewhat more broadly.

Definition 1 An array $a_{n i}$ is asymptotically mse-stationary if

$$
\frac{1}{n} \sum_{i=1}^{[n r]} a_{n i} a_{n i}^{\prime} \Rightarrow r A
$$

where $A=\lim _{n \rightarrow \infty} \frac{1}{n} \sum_{i=1}^{n} E\left(a_{n i} a_{n i}^{\prime}\right)$.

Intuitively, a process is asymptotically mse-stationary if its second moments are constant, while the behavior of higher-order moments is irrelevant. Strictly stationary, ergodic, and square integrable processes are asymptotically stationary, as are certain deterministic cyclic processes, seasonal dummies, and "stationary" forms of heteroskedasticity.

An interesting example is an $\mathrm{AR}(1)$ subject to small structural change:

$$
y_{n i}=\beta_{n i} y_{n i-1}+e_{n i}
$$

with $e_{n i}$ iid, and $\beta_{n i}$ following (2)-(3). Since $\beta_{n i}$ changes from $\beta$ to $\beta+\theta_{n}$ at time $t_{0}, y_{n i}$ is non-stationary. Yet since $\theta_{n} \rightarrow 0$ as $n \rightarrow \infty$, this non-stationarity may not be important in 
large samples. Indeed, we can calculate that as $n \rightarrow \infty$,

$$
E\left(\frac{1}{n} \sum_{i=1}^{[n r]} y_{n i}^{2}\right)=\sigma^{2} \frac{1}{n} \sum_{i=1}^{[n r]} \sum_{j=1}^{\infty} \beta_{n i+1-j}^{2} \rightarrow r A
$$

where $A=\sigma^{2} /\left(1-\beta^{2}\right)$. It follows by standard arguments that (8) holds for $y_{n i}$, so that $y_{n i}$ is asymptotically mse-stationary.

We now explore the distribution theory for our model under this assumption.

Theorem 1 If Assumption 1 holds, $x_{n i}$ is asymptotically mse-stationary, (4) holds, and for some $q>1$,

$$
\sup _{n \geq 1, i \leq n} E\left|x_{n i} e_{n i}\right|^{2 q}<\infty
$$

then the asymptotic null distributions of $\mathrm{SupF}_{n}, \mathrm{ExpF}_{n}, \mathrm{AveF}_{n}$, and $\mathrm{L}_{n}$ are those given in Andrews (1993), Andrews and Ploberger (1994) and Nyblom (1989).

Theorem 1 shows that the key assumption for the asymptotic theory is the asymptotic constancy of second moments. Intuitively, in linear regression information is reflected in the second moments of the data, and when the data are asymptotically mse-stationary information is accumulated linearly.

\subsection{Asymptotically Non-Stationary Processes}

The key condition for Theorem 1 is that the second moments of the cumulated data grow linearly. In this section we explore the consequences of violations of this assumption. We now consider a set of high-level conditions on the sample moments. We will give a list of standard examples which satisfy these conditions at the end of this section.

Assumption 2 As $n \rightarrow \infty$

$$
\begin{gathered}
\frac{1}{n} \sum_{i=1}^{[n r]} x_{n i} x_{n i}^{\prime} \Rightarrow M(r) \\
\frac{1}{n} \sum_{i=1}^{[n r]} x_{n i} x_{n i}^{\prime} e_{n i}^{2} / \sigma^{2} \Rightarrow V(r)
\end{gathered}
$$


and

$$
\frac{1}{\sigma \sqrt{n}} \sum_{i=1}^{[n r]} x_{n i} e_{n i} \Rightarrow N(r),
$$

where $M(r), V(r)$ and $N(r)$ are random $m \times m, m \times m$, and $m \times 1$ matrix processes, respectively, such that $M(r)$ and $V(r)$ are continuous in $r$ almost surely, for any $r>0$, $M(r)>0$ and $V(r)>0$ a.s., and conditionally on $\{V(s): 0 \leq s \leq 1\}, N(r)$ is a mean-zero Gaussian process with conditional covariance kernel

$$
E\left(N\left(r_{1}\right) N\left(r_{2}\right)^{\prime}\right)=V\left(\min \left(r_{1}, r_{2}\right)\right)
$$

Assumption 2 is a generalization of asymptotic mse-stationarity, because if $x_{n i}$ and $x_{n i} e_{n i}$ are asymptotically mse-stationary, (11)-(12)-(13) are satisfied with $M(r)$ and $V(r)$ linear matrix functionals, and $N(r)$ a Brownian motion (this is the essence of the proof of Theorem 1). Below, we discuss a set of examples which satisfy Assumption 2, and section 4 explores in detail a particular example. First we give the asymptotic distributions of the structural change tests under local alternatives.

Theorem 2 Under Assumptions 1 and 2,

$$
\begin{aligned}
F_{[n r]} & \Rightarrow\left(N^{*}(r)-Q(r) \delta\right)^{\prime} M^{*}(r)^{-1}\left(N^{*}(r)-Q(r) \delta\right) \\
& \equiv F(r \mid \delta)
\end{aligned}
$$

where

$$
\begin{gathered}
N^{*}(r)=N(r)-M(r) M(1)^{-1} N(1) \\
Q(r)=\left(M(r)-M(r) M(1)^{-1} M\left(\tau_{0}\right)\right)-\left(M(r)-M\left(\tau_{0}\right)\right) I\left(r \geq \tau_{0}\right),
\end{gathered}
$$

and

$$
M^{*}(r)=M(r)-M(r) M(1)^{-1} M(r) .
$$

Hence

1. $\operatorname{SupF}_{n} \rightarrow_{d} \sup _{\pi_{1} \leq r \leq \pi_{2}} F(r \mid \delta)$;

2. $\operatorname{ExpF}_{n} \rightarrow_{d} \ln \left[\frac{1}{\pi_{2}-\pi_{1}} \int_{\pi_{1}}^{\pi_{2}} \exp \left(\frac{1}{2} F(r \mid \delta)\right) d r\right]$; 
3. $\mathrm{AveF}_{n} \rightarrow_{d} \frac{1}{\pi_{2}-\pi_{1}} \int_{\pi_{1}}^{\pi_{2}} F(r \mid \delta) d r$

4. $\mathrm{L}_{n} \rightarrow{ }_{d} \int_{0}^{1}\left(N^{*}(r)-Q(r) \delta\right)^{\prime} M(1)^{-1}\left(N^{*}(r)-Q(r) \delta\right) d r$.

Theorem 2 gives the asymptotic distributions under local departures from $H_{0}$. To find the null distribution, set $\delta=0$, in which case we find

$$
F(r) \equiv F(r \mid 0)=N^{*}(r)^{\prime}\left(M(r)-M(r) M(1)^{-1} M(r)\right)^{-1} N^{*}(r) .
$$

Unless $M(r)$ is linear in $r, N^{*}(r)$ will not be a Brownian bridge and $F(r)$ will not equal the squared tied-down Bessell process that appears in Andrews (1993). It follows that the asymptotic distributions of the test statistics given in Theorem 2 are different than the distributions tabulated by Andrews (1993), Andrews and Ploberger (1994) and Nyblom (1989). Apparently, these tests for structural change are not asymptotically pivotal when we allow for asymptotic non-stationarity ${ }^{1}$.

The null sampling distributions of the test statistics examined in Theorem 2 appear to depend on the functions $M(r)$ and $V(r)$. No other nuisance parameters enter the asymptotic distributions. Under the alternative hypothesis $(\delta \neq 0)$, the only additional nuisance parameter is $\tau_{0}$, the true timing of the structural change in regression parameter.

Theorem 2 applies in many interesting examples. In the following examples, for simplicity we assume that the error $e_{n i}$ satisfies (4).

Example 1: Linear Trend: $x_{n i}=i / n$. Then $M(r)=r^{3} / 3$ and $N(r)=\int_{0}^{r} s d W(s)$.

Example 2: Trend in Variance: $x_{n i}=\sqrt{i / n} v_{i}$ with $v_{i} \operatorname{iid}(0,1)$. Then $M(r)=r^{2} / 2$ and $N(r)=\int_{0}^{r} s^{1 / 2} d W(s)$.

Example 3: Stochastic Trend: $x_{n i}=n^{-1 / 2} z_{i}$, where $\Delta z_{i}$ is $I(0)$ and independent of $e_{n i+j}$ for all $j$. Then letting $W_{2}$ and $W_{1}$ denote independent Brownian motions, $M(r)=\int_{0}^{r} W_{2}(s)^{2} d s$ and $N(r)=\int_{0}^{r} W_{2}(s) d W_{1}(s)$. Using similar reasoning, Assumption 2 applies to cointegrated regression models estimated using the leads-and-lags technique of Saikkonen (1991), Phillips and Loretan (1991) and Stock and Watson (1993).

\footnotetext{
${ }^{1}$ The results of Theorem 2 stand in stark contrast to those for the Chow (1960) test for structural change of known timing. From (15), one can deduce that the asymptotic null distribution of the Chow F statistic is chi-square and hence it is asymptotically pivotal.
} 
In all three examples, $M(r)$ is non-linear and $N(r)$ is not a Brownian motion. This nonlinearity implies that the tabulated critical values in Andrews (1993), Andrews and Ploberger (1994) and Nyblom (1989) will be inappropriate in these contexts. We now turn to a more thorough examination of a particular example of interest.

\section{Structural Change in the Marginal Distribution}

To illustrate the possible divergence between the distribution results of Theorem 1 and Theorem 2, we examine the case where there is a single structural change in the marginal distribution of $x_{n i}$ at date $k$. We extend Definition 1 slightly by saying that $a_{i}$ is asymptotically msestationary over the region $\left(k_{1}, k_{2}\right)$ with $k_{1}=\left[n \kappa_{1}\right], k_{2}=\left[n \kappa_{2}\right]$ if $\frac{1}{n} \sum_{i=k_{1}}^{k_{2}} a_{i} a_{i}^{\prime} \Rightarrow\left(\kappa_{2}-\kappa_{1}\right) A_{12}$ for some matrix $A_{12}$.

Assumption 3 Let $k=[n \kappa]$ and $\kappa \in(0,1)$. The variables $x_{n i}$ is asymptotically msestationary for $i<k$, and $x_{n i}$ is asymptotically mse-stationary for $i \geq k$. (4) and (10) hold.

Assumption 3 specifies that the marginal distribution of $x_{t}$ is asymptotically stationary before and after the date $k$, but allows an arbitrary structural change at observation $k$. The change may occur in the mean, variance and/or serial correlation in the regressor. Note that Assumption 3 allows for lagged dependent variables as discussed in section 3.1. It is straightforward to verify that Assumption 3 implies Assumption 2 with

$$
M(r)=M_{1} r+\left(M_{2}-M_{1}\right)(r-\kappa) I(r \geq \kappa)
$$

and $V(r)=M(r)$, where

$$
M_{1}=\lim _{n \rightarrow \infty} \frac{1}{n \kappa} \sum_{i=1}^{[n \kappa]-1} E\left(x_{n i} x_{n i}^{\prime}\right)
$$

and

$$
M_{2}=\lim _{n \rightarrow \infty} \frac{1}{n(1-\kappa)} \sum_{i=[n \kappa]}^{n} E\left(x_{n i} x_{n i}^{\prime}\right) .
$$

Since Assumption 2 holds, the distribution theory of Theorem 2 applies. 
Under Assumption 3 the function $M(r)$ is a piece-wise linear function in $r$ with a kink at $r=\kappa$. We see that the accumulation of information is non-linear, rather than linear.

To illustrate the impact this non-linearity has on the asymptotic distributions, we consider the leading case of one regressor $(m=1)$ so that $M_{2}$ and $M_{1}$ are scalars. For this case we can make some interesting analytical observations about the asymptotic null distributions of the SupF and L statistics. Set $\omega=M_{2} / M_{1}-1$ and

$$
v(r)=\frac{r+\omega(r-\kappa) I(r \geq \kappa)}{1+\omega(1-\kappa)} .
$$

Let

$$
\operatorname{SupF}=\sup _{\pi_{1} \leq r \leq \pi_{2}} \frac{N^{*}(r)^{2}}{M^{*}(r)}
$$

denote the asymptotic null distribution of $\mathrm{SupF}_{n}$ under Assumption 3 (from Theorem 2), where $N^{*}(r), M^{*}(r)$ and $M(r)$ are defined in (16), (17) and (18), respectively.

\section{Theorem 3}

$$
\operatorname{SupF} \equiv \sup _{\pi_{1}^{*} \leq r \leq \pi_{2}^{*}} \frac{W^{*}(r)^{2}}{r(1-r)}
$$

where $W^{*}(r)$ is a standard Brownian bridge, $\pi_{1}^{*}=v\left(\pi_{1}\right)$, and $\pi_{2}^{*}=v\left(\pi_{2}\right)$.

The distribution in Theorem 3 is identical to that found by Andrews (1993) for stationary processes, but depends on the index

$$
\lambda^{*}=\frac{\pi_{2}^{*}\left(1-\pi_{1}^{*}\right)}{\pi_{1}^{*}\left(1-\pi_{2}^{*}\right)}
$$

rather than $\lambda=\pi_{2}\left(1-\pi_{1}\right) /\left[\pi_{1}\left(1-\pi_{2}\right)\right]$ as found by Andrews. Thus the relevant measure of "spread" between $\pi_{1}$ and $\pi_{2}$ is not based on the linear measure $r$ implicit in the definition of $\lambda$, but should be based on the non-linear measure $v(r)$ reflecting the actual rate of accumulation of sample information. Note that $\lambda^{*}$ is a function only of $\pi_{1}^{*}$ and $\pi_{2}^{*}$, the "relative information" cumulated in the regressors at the times $\pi_{1}$ and $\pi_{2}$.

From Table 1 of Andrews (1993), we see that the asymptotic critical values for $\operatorname{SupF}_{n}$ are increasing in $\lambda$. Hence if $\lambda^{*}>\lambda$ the statistic $\operatorname{SupF}_{n}$ will tend to reject too frequently if Andrews' critical values are used. On the other hand, if $\lambda^{*}<\lambda$, then $\operatorname{SupF}_{n}$ will tend to reject too infrequently, reducing power. Setting $\pi_{1}=.15$ and $\pi_{2}=.85$, Figure 1 plots $\lambda^{*}$ as 
a function of $\kappa$ for four positive values of $\omega$. For each $\omega, \lambda^{*}$ is maximized at $\kappa=\pi_{1}$ and minimized at $\kappa=\pi_{2}$. As $\omega$ increases, $\lambda^{*}$ can become arbitrarily large. The sampling implication is that the Quandt-Andrews $\mathrm{SupF}_{n}$ statistic can have arbitrarily large size distortion.

While we cannot find a general analytic expression for the L distribution, we can find its limiting behavior for large $\omega$. Again for $m=1$ let

$$
\mathrm{L}(\omega, \kappa)=\frac{\int_{0}^{1} N^{*}(r)^{2} d r}{M(1)}
$$

denote the asymptotic null distribution of $\mathrm{L}_{n}$ under Assumption 3.

\section{Theorem 4}

$$
\operatorname{plim}_{\omega \rightarrow \infty} \mathrm{L}(\omega, \kappa)=(1-\kappa) \int_{0}^{1} W^{*}(r)^{2},
$$

where $W^{*}(r)$ is a standard Brownian bridge.

Note that the conventional Nyblom asymptotic distribution is $\mathrm{L}(0, \kappa) \equiv \int_{0}^{1} W^{*}(r)^{2}$. Theorem 4 shows that if $\omega$ is large, then the Nyblom statistic will be asymptotically conservative, in the sense that the asymptotic distribution of the test statistic will be dominated by the conventional distribution. This suggests that Nyblom's test will substantially underreject the null hypothesis when $\omega$ and $\kappa$ are large.

Figure 2 plots numerical estimates ${ }^{2}$ of the asymptotic Type I error ${ }^{3}$ (at the $10 \%$ nominal level) for the four asymptotic distributions (SupF, ExpF, AveF, and L) as a function of $\omega$, for $\kappa=.25, .50, .75$, and $.95^{4}$. The $\mathrm{SupF}_{n}, \mathrm{ExpF}_{n}$, and AveF $n$ tests set $\pi_{1}=.15$ and $\pi_{2}=.85$. The most striking feature of the plots is that the Nyblom test substantially underrejects for large $\omega$ (as predicted by Theorem 4). The second most noticeable feature is that $\operatorname{SupF}_{n}$ over-rejects for $\kappa=.25$ and under-rejects for $\kappa=.95$ (as predicted by Theorem 3). A very interesting finding is that the Andrews-Ploberger $\operatorname{ExpF}_{n}$ test has virtually zero size distortion for any $\kappa$ and $\omega$.

Figure 3 plots estimated ${ }^{5}$ asymptotic local power functions for the four test statistics (not size-corrected) for the case of a large structural change in the marginal distribution,

\footnotetext{
${ }^{2}$ Generated using random normal samples of size 1000 with 50,000 replications for each $\omega=1,2, \ldots, 16$.

${ }^{3}$ Rejection rates using asymptotic critical values and fixed parameters.

${ }^{4}$ Only graphs for positive $\omega$ are shown as they are symmetric in the transformation $(\omega, \kappa) \rightarrow(-\omega, 1-\kappa)$.

${ }^{5}$ Generated using random normal samples of size 1000 with 20,000 replications for $\delta=1,2, \ldots, 10$.
} 
$\omega=10$. The plots are as a function of $\delta$, for $\kappa=.25, .50, .75$, and .95 , and $\tau_{0}$ is set to equal $\kappa$. The plots show that for $\kappa \leq .75$, the power of the Nyblom test is adversely affected by the shift in the marginal distribution. In the case $\kappa=.25$, the power loss is dramatic. The AveF test also suffers a mild power loss in some cases. In contrast, the ExpF and SupF tests appear to have the best power, with the exception of the case $\kappa=.95$ (where the Nyblom test is over-sized).

In summary, the numerical analysis of the asymptotic distributions of the test statistics suggest that at least for $m=1$ the Nyblom test is quite poorly behaved in the presence of shifts in the marginal distribution, yet the Andrews-Ploberger $\operatorname{ExpF}_{n}$ statistic is essentially unaffected by such shifts. The popular Quandt-Andrews $\operatorname{SupF}_{n}$ statistic suffers from a mild size distortion, but with no noticeable effect on power.

\section{$5 \quad$ Bootstrapping}

\subsection{The Fixed Regressor Bootstrap}

We have seen in the previous sections that non-stationarity or structural change in the marginal distribution affects the asymptotic distributions of the test statistics in complicated ways. An alternative is to consider a bootstrap distribution. The term "bootstrap" was introduced by Efron (1989) and has since spawned a large literature. Most of the theory and techniques require random samples. Extensions to dependent data have been confined to strictly stationary processes, including the parametric bootstrap of Bose (1988) and the block resampling bootstraps of Carlstein (1986) and Künsch (1989). To my knowledge, there is no theoretical literature ${ }^{6}$ which applies to the present context - involving non-standard test statistics and explicitly non-stationary data processes. A priori, it is not clear if bootstrap methods will work, as we know that standard bootstrap techniques fail in the context of non-stationary autoregressions, see Basawa, et. al. (1991).

In our model, appropriate application of the bootstrap is not obvious. Under Assumption $2, x_{n i}$ need not be stationary, so block re-sampling is inappropriate. Parametric bootstrap

\footnotetext{
${ }^{6}$ In an interesting contribution, Diebold and Chen (1996) provide simulation evidence (but no theory) that the parametric bootstrap works well for structural change tests applied to AR(1) processes.
} 
methods are also inappropriate in conditional regression models (except in the special case in which $x_{n i}$ is strictly exogenous). We wish to avoid methods which require the joint modelling of $y_{n i}$ and $x_{n i}$ which require the correct specification of the marginal distribution (including any structural changes). Such modelling violates the principle of the regression model, where the goal is to condition on the regressors, and hence ignore their marginal distribution.

Despite these concerns, we are able to successfully employ what we call the "Fixed Regressor Bootstrap," which treats the regressors $x_{n i}$ as if they are fixed (exogenous) even when they contain lagged dependent variables. The discussion which follows is for the SupF test, yet the method applies as well for the other tests. There are two forms of the fixed regressor bootstrap introduced here, one appropriate if the error $e_{n i}$ is homoskedastic (4) and the other appropriate under heteroskedasticity.

For the homoskedastic bootstrap, let $\left\{y_{n i}(b): i=1, \ldots, n\right\}$ be a random sample from the $N(0,1)$ distribution. Regress $y_{n i}(b)$ on $x_{n i}$ to get residual variance $\hat{\sigma}^{2}(b)$ and regress $y_{n i}(b)$ on $x_{n i}$ and $x_{n i} I(i \leq t)$ to get the residual variance $\hat{\sigma}_{t}^{2}(b)$ and Wald sequence

$$
F_{t}(b)=\frac{(n-m) \hat{\sigma}^{2}(b)-(n-2 m) \hat{\sigma}_{t}^{2}(b)}{\hat{\sigma}_{t}^{2}(b)} .
$$

The bootstrap test statistic is $\operatorname{SupF}_{n}(b)=\sup _{t_{1} \leq t \leq t_{2}} F_{t}(b)$. Let $G_{n}(x)=P\left(\operatorname{SupF}_{n}(b) \leq x \mid \Im_{n}\right)$ denote the conditional distribution function of $\operatorname{SupF}_{n}(b)$ (conditional on the data). The bootstrap p-value is $p_{n}=1-G_{n}\left(\mathrm{SupF}_{n}\right)$. The bootstrap test rejects $H_{0}$ when $p_{n}$ is small.

We can allow for heteroskedastic errors by making a small modification. Set $y_{n i}^{h}(b)=$ $u_{i}(b) \tilde{e}_{i}$, where $\left\{u_{i}(b): i=1, \ldots, n\right\}$ is an iid $N(0,1)$ sample and $\tilde{e}_{i}$ are the regression residuals defined in section 2. Construct the bootstrap test statistic $\operatorname{SupF}_{n}^{n}(b)$ as before, substituting $y_{n i}^{h}(b)$ for $y_{n i}(b)$ in the previous paragraph. This has the bootstrap distribution $G_{n}^{h}(x)=$ $P\left(\operatorname{SupF}_{n}^{h}(b) \leq x \mid \Im_{n}\right)$ from which we define the bootstrap p-value $p_{n}^{h}=1-G_{n}^{h}\left(\operatorname{SupF}_{n}\right)$.

While $G_{n}(\cdot)$ is unknown, it may be calculated by simulation. Let $\operatorname{SupF}_{n}(j), j=1, \ldots, J$ denote (conditionally) independent draws from the distribution $\operatorname{SupF}_{n}(b)$. The simulated bootstrap p-value $p_{n}(J)$ is then calculated by counting the percentage of simulated bootstrap test statistics $\operatorname{SupF}_{n}(j)$ which exceed the sample value $\operatorname{SupF}_{n}$. As $J \rightarrow \infty, p_{n}(J) \rightarrow p_{n}$ almost surely, so the error due to simulation can be made arbitrarily small. For the case of heteroskedastic errors, a simulation estimate of $p_{n}^{h}$, denoted $p_{n}^{h}(J)$ may be constructed 
similarly. We follow the bootstrap literature (see Hall (1994)) and consider $p_{n}$ and $p_{n}^{h}$ to be the bootstrap statistics of interest. It is important to remember, however, that inference in practice is based on the randomized estimates $p_{n}(J)$ or $p_{n}^{h}(J)$, which may induce additional error unless $J$ is large.

\section{$5.2 \quad$ Asymptotic Theory}

The following notation will be helpful. Let $T(\delta)=\sup _{\pi_{1} \leq r \leq \pi_{2}} F(r \mid \delta)$ so that by Theorem 2, $\operatorname{SupF}_{n} \rightarrow{ }_{d} T(\delta)$ and $T(0)$ denotes the null distribution. Let $G(x)=P(T(0) \leq x)$ denote the null asymptotic distribution function, and define the random variable $p(\delta)=1-G(T(\delta))$. Note that the distribution of $p(0)$ is $U[0,1]$.

Let " $\Rightarrow_{p}$ " denote weak convergence in probability as defined by Gine and Zine (1990). The concept "weak convergence in probability" generalizes convergence in distribution to allow for conditional (i.e. random) distribution functions. This is necessary for bootstrap theory as the empirical distribution used for re-sampling is data-dependent. We first state the results for the homoskedastic bootstrap.

Theorem 5 Under Assumptions 1, 2, and (4), $\operatorname{SupF}_{n}(b) \Rightarrow_{p} T(0)$ and $p_{n} \rightarrow_{d} p(\delta)$.

Corollary 1 Under Assumptions 1, 2, (4), and $H_{0}, p_{n} \rightarrow_{d} U[0,1]$.

The first result of Theorem 5 states that the conditional distribution function $G_{n}(\cdot)$ is close to $G(\cdot)$ if $n$ is sufficiently large. This means that p-value calculations based on $G_{n}$ are asymptotically equivalent to those based on $G$. The second result of Theorem 5 gives the asymptotic distribution of the bootstrap p-value $p_{n}$. In particular, we find in Corollary 1 that under $H_{0}, p_{n}$ is asymptotically distributed $U[0,1]$, which is pivotal, so the nuisance parameter problem has been solved (for large samples). We now state the result for the heteroskedastic bootstrap.

Theorem 6 Under Assumptions 1 and $2, \operatorname{SupF}_{n}^{n}(b) \Rightarrow_{p} T(0)$ and $p_{n}^{h} \rightarrow_{d} p(\delta)$.

Corollary 2 Under Assumptions 1, 2, and $H_{0}, p_{n}^{h} \rightarrow_{d} U[0,1]$. 
Theorem 6 and Corollary 2 show that the heteroskedastic bootstrap achieves the correct asymptotic distribution under general forms of conditional heteroskedasticity. This holds even though $\mathrm{SupF}_{n}$ has not been constructed to allow for heteroskedasticity.

It is also possible to derive the asymptotic local power functions of the bootstrap tests from Theorems 5 and 6, which allows us to assess the behavior of the tests under (local) alternatives. From Theorem 5, we can calculate that

$$
\begin{aligned}
\pi_{a}(\delta) & =\lim _{n \rightarrow \infty} P\left(p_{n} \leq a\right) \\
& =P(p(\delta) \leq a) \\
& =P(T(\delta)>b)
\end{aligned}
$$

where $b=G^{-1}(a)$. The function $\pi_{a}(\delta)$ is the asymptotic local power function. As $|\delta| \rightarrow \infty$ the non-centrality effect in $F(r \mid \delta)$ causes $T(\delta) \rightarrow \infty$ almost surely. Hence for any $a$, $\pi_{a}(\delta) \rightarrow 1$ as $|\delta| \rightarrow \infty$. In other words, the asymptotic local power function is increasing in $|\delta|$, and the asymptotic probability of rejecting the null hypothesis can be made arbitrarily high by selecting a sufficiently large $\delta$.

This argument does not prove that the test is consistent against fixed alternatives, however, as $\theta_{n}$ fixed and independent of $n$ is not covered by our theory. A formal proof of consistency against fixed alternatives appears to be quite intricate, and will not be attempted in this paper.

\subsection{Small Sample Distributions}

To investigate the performance of our bootstrap tests in a small sample, we report a limited Monte Carlo experiment. The regression model is a single equation from a bi-variate VAR:

$$
y_{i}=\alpha_{0}+\alpha_{1} y_{i-1}+\alpha_{2} y_{i-2}+\alpha_{3} y_{i-3}+\beta_{1} x_{i-1}+\beta_{2} x_{i-2}+\beta_{3} x_{i-3}+e_{i}
$$

with a sample size of $n=50$. We set $\alpha_{1}=.5$ and $\beta_{1}=1$ while the other regression parameters are set to zero.

Seven models for the regressors $x_{i}$ are considered. Below, let $\eta_{i}$ be iid student $\mathrm{t}$ with 5 degrees of freedom, let $u_{i}$ be iid $N(0,1)$, and let $c_{i}=1+2 i / n$. 
- IID: $x_{i}=\eta_{i}$.

- Mean Break: $x_{i}=\eta_{i}$ for $i \leq 25$ and $x_{i}=5+\eta_{i}$ for $i>25$.

- Variance Break: $x_{i}=\eta_{i}$ for $i \leq 25$ and $x_{i}=5 \eta_{i}$ for $i>25$.

- Mean Trend: $x_{i}=c_{i}+u_{i}$.

- Variance Trend: $x_{i}=\sqrt{c_{i}} u_{i}$.

- Stochastic Trend: $x_{i}=x_{i-1}+u_{i}$.

- Stochastic Variance: $x_{i}=\left(\sum_{j=1}^{j} w_{i}\right) u_{i}$, where $w_{i}$ is iid $N(0,1)$.

Two specifications for the regression error $e_{i}$ are considered. The first is that $e_{i}$ is iid student $\mathrm{t}$ with 5 degrees of freedom. The second is that $e_{i}$ is conditionally heteroskedastic $e_{i} \sim N\left(0,1+.25 x_{i-1}^{2}\right)$.

The random regressors $x_{i}$ are independently generated for each Monte Carlo replication. In all experiments, the number of bootstrap replications is $J=1000$ and the number of simulation replications is 5000 . We report results for tests of nominal size $10 \%$.

We restrict attention to the SupF statistic of Andrews (1993) for simplicity. Three distributional approximations are considered. The first uses the asymptotic approximation of Andrews (1993), the second uses the homoskedastic fixed regressor bootstrap, and the third the heteroskedastic fixed regressor bootstrap. In all experiments, the null hypothesis of coefficient stability holds, so the rejection rate should be ideally $10 \%$.

The results are summarized in Table 1. First consider the case of homoskedastic errors, reported in the first half of the Table. In all cases, tests based on the conventional asymptotic approximation substantially over-reject. Tests based on the homoskedastic bootstrap have better size, and tests based on the heteroskedastic bootstrap are close to the correct rejection frequency.

The second half of the table reports results for the heteroskedastic error process. The performance of all test statistic deteriorates significantly. Tests based on the conventional asymptotic approximation are extremely poor. Tests based on the homoskedastic bootstrap are not much improved, which should not be surprising since the technique is not designed 
Table 1: Small Sample Type I Error, 10\% Nominal Size

\begin{tabular}{|l|c|c|c|c|c|c|c|c|}
\hline & \multicolumn{7}{|c|}{ Regressor Process } \\
\hline & IID & $\begin{array}{c}\text { Mean } \\
\text { Break }\end{array}$ & $\begin{array}{c}\text { Variance } \\
\text { Break }\end{array}$ & $\begin{array}{c}\text { Mean } \\
\text { Trend }\end{array}$ & $\begin{array}{c}\text { Variance } \\
\text { Trend }\end{array}$ & $\begin{array}{c}\text { Stochastic } \\
\text { Mean }\end{array}$ & $\begin{array}{c}\text { Stochastic } \\
\text { Variance }\end{array}$ \\
\hline \multicolumn{8}{|c|}{ Homoskedastic Regression Error } \\
\hline Asymptotic Distribution & 16 & 21 & 21 & 19 & 18 & 22 & 21 \\
Homoskedastic Bootstrap & 12 & 14 & 14 & 13 & 12 & 15 & 15 \\
Heteroskedastic Bootstrap & 10 & 9 & 7 & 10 & 09 & 10 & 10 \\
\hline \multicolumn{8}{|c|}{ Heteroskedastic Regression Error } \\
\hline Asymptotic Distribution & 21 & 43 & 71 & 22 & 18 & 40 & 50 \\
Homoskedastic Bootstrap & 14 & 33 & 64 & 15 & 12 & 31 & 42 \\
Heteroskedastic Bootstrap & 10 & 19 & 34 & 11 & 8 & 20 & 23 \\
\hline
\end{tabular}

Note: Rejection Frequencies (\%) in 5000 Replications. Standard Error of Estimates $=0.4$

to be robust to heteroskedasticity. The heteroskedastic bootstrap works much better than the other tests for most regressor processes, achieving correct size in three of the seven cases. In the most extreme cases, however, the heteroskedastic bootstrap also over-rejects relative to the nominal size.

The simulation results show that the fixed regressor bootstrap improves on asymptotic approximations, but does not completely solve the inference problem.

\section{Conclusion}

This paper has attempted a careful examination of modern tests for structural change and the associated asymptotic distribution theory. We argue that the assumption that the regressors are stationary (without structural change) is inappropriate in the context of testing for structural change in a regression, and that relaxing this assumption has consequences for the asymptotic theory. We found that the Nyblom L statistic is sensitive to this assumption, so is generally not recommended for empirical application in regression models. In a numerical study, the Andrews-Ploberger exponentially-weighted ExpF statistic appears to be the most stable with respect to structural change in the marginal equation.

We show that correct asymptotic inference may be obtained from a bootstrap distri- 
bution. We consider a simple fixed regressor bootstrap, which treats the right-hand-side regressors as fixed (even the lagged dependent variables). This produces the correct asymptotic distribution under a wide range of conditions, such as arbitrary structural change in the regressors including multiple structural breaks, time trends and certain stochastic trends. The regressors need not be strictly exogenous and the regression errors may be conditionally heteroskedastic. This procedure is computationally cheap and easy to program. In many contexts, bootstrap techniques can improve on the first-order asymptotic distribution when they approximate an Edgeworth correction (see Hall, 1994). This appears unlikely in the case of the fixed regressor bootstrap. Other bootstrap procedures are conceivable, but care must be taken to correctly mimic the distribution under the null hypothesis, and not distort the possible non-stationarity in the conditioning variables.

A GAUSS program which implements the empirical techniques discussed in this paper is available upon request from the author or his web homepage.

\section{Appendix: Mathematical Proofs}

Proof of Theorem 1. It is well known that

$$
F_{t}=\frac{\hat{S}_{t}^{\prime}\left(M_{t}-M_{t} M_{n}^{-1} M_{t}\right)^{-1} \hat{S}_{t}}{\hat{\sigma}_{t}^{2} / \sigma^{2}}
$$

where $M_{t}=n^{-1} \sum_{i=1}^{t} x_{n i} x_{n i}^{\prime}$ and $\hat{S}_{t}=\left(\sigma^{2} n\right)^{-1 / 2} \sum_{i=1}^{t} x_{n i} \hat{e}_{i}$. Under $H_{0}, \hat{S}_{t}=S_{t}-M_{t} M_{n}^{-1} S_{n}$, where $S_{t}=\left(\sigma^{2} n\right)^{-1 / 2} \sum_{i=1}^{t} x_{n i} e_{n i}$. By the asymptotic mse-stationarity of $x_{n i}, M_{[n r]} \Rightarrow r M$ where $M=\lim _{n \rightarrow \infty} n^{-1} \sum_{i=1}^{n} E\left(x_{n i} x_{n i}^{\prime}\right)$. We next show that for any $\alpha \neq 0$,

$$
\alpha^{\prime} \frac{1}{n} \sum_{i=1}^{[n r]} x_{n i} x_{n i}^{\prime} e_{n i}^{2} \alpha \Rightarrow r \alpha^{\prime} M \alpha \sigma^{2}
$$

Let $\xi_{n i}=\alpha^{\prime} x_{n i} x_{n i}^{\prime} \alpha\left(e_{n i}^{2}-\sigma^{2}\right)$. By (4), $\xi_{n i}$ is a martingale difference array. Thus for any fixed $r$, by Burkholder's inequality (e.g. Hall and Heyde (1980), p. 23)), for some $C<\infty$,

$E\left|\frac{1}{n} \sum_{i=1}^{[n r]} \xi_{n i}\right|^{q} \leq\left.\left. C \frac{1}{n^{q}} E\left|\sum_{i=1}^{[n r]}\right| \xi_{n i}\right|^{2}\right|^{q / 2} \leq C \frac{1}{n^{q}} \sum_{i=1}^{[n r]} E\left|\xi_{n i}\right|^{q} \leq 2\left(\alpha^{\prime} \alpha\right)^{q} C n^{1-q} \sup _{n \geq 1, i \leq n} E\left|x_{n i} e_{n i}\right|^{2 q} \rightarrow 0$ 
by (10). By Markov's inequality it follows that $\frac{1}{n} \sum_{i=1}^{[n r]} \xi_{n i} \rightarrow_{p} 0$. Thus pointwise in $r$,

$$
\alpha^{\prime} \frac{1}{n} \sum_{i=1}^{[n r]} x_{n i} x_{n i}^{\prime} e_{n i}^{2} \alpha=\alpha^{\prime} M_{[n r]} \sigma^{2} \alpha+\frac{1}{n} \sum_{i=1}^{[n r]} \xi_{n i} \rightarrow_{p} r \alpha^{\prime} M \alpha \sigma^{2} .
$$

This convergence is also uniform over $0 \leq r \leq 1$, since the left-hand argument of (24) is monotonically increasing in $r$ and the limit function is continuous in $r$ (for a proof, see Lemma A.9 of Hansen (1998)). This establishes (24).

Take any $\eta>0$, set $K=\operatorname{tr} M \sigma^{2} / \eta$ and $r_{k}=k / K$ for $k=0, \ldots, K$. By (24),

$$
\max _{i \leq n} \frac{1}{n} x_{n i}^{\prime} x_{n i} e_{n i}^{2} \leq \max _{k \leq K} \sum_{i=\left[n r_{k}\right]+1}^{\left[n r_{k+1}\right]} \frac{1}{n} x_{n i}^{\prime} x_{n i} e_{n i}^{2} \rightarrow_{p} \operatorname{tr} M \sigma^{2} / K=\eta .
$$

Since $x_{n i} e_{n i}$ is a martingale difference sequence, (24) and (25) imply that $S_{[n r]} \Rightarrow B(r)$, a vector Brownian motion with covariance matrix $M$, see Davidson (1994), Theorem 27.14. It is not hard to show that $\hat{\sigma}_{[n r]}^{2} \Rightarrow \sigma^{2}$. The stated results follow by standard manipulations.

Proof of Theorem 2. Under $H_{1}$ (see (3) and (5)) we can calculate that

$$
\begin{aligned}
\hat{S}_{t} & =\frac{1}{\sigma \sqrt{n}} \sum_{i=1}^{t} x_{n i} \hat{e}_{i} \\
& =S_{t}-M_{t} M_{n}^{-1} S_{n}+\left(M_{t}-M_{t_{0}}\right) I\left(t \geq t_{0}\right) \delta-M_{t} M_{n}^{-1}\left(M_{n}-M_{t_{0}}\right) \delta
\end{aligned}
$$

Thus

$$
\begin{aligned}
\hat{S}_{[n r]} \Rightarrow & N(r)-M(r) M(1)^{-1} N(1)+\left(M(r)-M\left(\tau_{0}\right)\right) I\left(r \geq \tau_{0}\right) \delta \\
& -\left(M(r)-M(r) M(1)^{-1} M\left(\tau_{0}\right)\right) \delta \\
= & N^{*}(r)-Q(r) \delta,
\end{aligned}
$$

and

$$
\begin{aligned}
F_{[n r]} & =\frac{\hat{S}_{[n r]}^{\prime}\left(M_{[n r]}-M_{[n r]} M_{n}^{-1} M_{[n r]}\right)^{-1} \hat{S}_{[n r]}}{\hat{\sigma}_{[n r]}^{2} / \sigma^{2}} \\
& \Rightarrow\left(N^{*}(r)-Q(r) \delta\right)^{\prime}\left(M(r)-M(r) M(1)^{-1} M(r)\right)^{-1}\left(N^{*}(r)-Q(r) \delta\right)
\end{aligned}
$$

by the continuous mapping theorem. The results for the test statistics follow from standard manipulations. 


\section{Proof of Theorem 3.}

Note that $M(r)=[1+\omega(1-\kappa)] v(r)$ where $v(r)$ is defined in (21). WLOG, rescaling allows us to set $M(r)=v(r)$. Let

$$
\phi(s)=(1+\omega(1-\kappa)) s-\frac{\omega}{1+\omega}((1+\omega(1-\kappa)) s-\kappa)^{+} .
$$

One can verify that $\phi(s)$ is monotonically increasing and $v(\phi(s))=s$. Thus $M(\phi(s))=s$, and $M^{*}(\phi(s))=s(1-s)$. Further, $W(s)=N(\phi(s))$ is a standard Brownian motion and $W^{*}(s)=N^{*}(\phi(s))$ is a Brownian bridge. Making the change of variables $r=\phi(s)$, we conclude that

$$
\sup _{\pi_{1} \leq r \leq \pi_{2}} \frac{N^{*}(r)^{2}}{M^{*}(r)}=\sup _{\pi_{1} \leq \phi(s) \leq \pi_{2}} \frac{N^{*}(\phi(s))^{2}}{M^{*}(\phi(s))}=\sup _{v\left(\pi_{1}\right) \leq s \leq v\left(\pi_{2}\right)} \frac{W^{*}(s)^{2}}{s(1-s)}
$$

Proof of Theorem 4. As in the proof of Theorem 3, rescale so that $M(r)=v(r)$. Note $v(1)=1$. For the change-of-variables $r=\phi(s)$ defined in $(27)$, the Jacobian is

$$
J(s)= \begin{cases}1+\omega(1-\kappa) & s<\kappa^{*} \\ \frac{1+\omega(1-\kappa)}{1+\omega} & s \geq \kappa^{*}\end{cases}
$$

where $\kappa^{*}=\kappa /(1+\omega(1-\kappa))$. As $\omega \rightarrow \infty, \kappa^{*} \rightarrow 0$ and

$$
\begin{aligned}
\frac{\int_{0}^{1} N^{*}(r)^{2} d r}{M(1)} & =\int_{0}^{1} N^{*}(\phi(s))^{2} J(s) d s \\
& =(1+\omega(1-\kappa)) \int_{0}^{\kappa^{*}} W^{*}(s)^{2} d s+\frac{(1+\omega(1-\kappa))}{1+\omega} \int_{\kappa^{*}}^{1} W^{*}(s)^{2} d s \\
& \rightarrow p p(1-\kappa) \int_{0}^{1} W^{*}(s)^{2} d s .
\end{aligned}
$$

Proof of Theorem 5. Define

$$
S_{t}(b)=\frac{1}{n} \sum_{i=1}^{t} x_{n i} u_{i}(b)
$$

Since $u_{i}(b)$ is iid $N(0,1)$, the stochastic process $S_{[n r]}^{b}$, conditional on the sample, has an exact distribution as a mean-zero Gaussian process with covariance kernel $M_{[n \min (r, s)]} \Rightarrow$ 
$M(\min (r, s))=V(\min (r, s))$ under $(4)$. Hence $S_{[n r]}(b) \Rightarrow_{p} N(r)$. Thus

$$
\begin{aligned}
F_{t}(b) & =\left(S_{t}(b)-M_{t} M_{n}^{-1} S_{n}(b)\right)^{\prime}\left(M_{t}-M_{t} M_{n}^{-1} M_{t}\right)^{-1}\left(S_{t}(b)-M_{t} M_{n}^{-1} S_{n}(b)\right) /\left(\hat{\sigma}_{t}^{2}(b) / \sigma^{2}\right) \\
& \Rightarrow p\left(N(r)-M(r) M(1)^{-1} N(1)\right)^{\prime} M^{*}(r)^{-1}\left(N(r)-M(r) M(1)^{-1} N(1)\right) \\
& \equiv F(r \mid 0)
\end{aligned}
$$

and

$$
\operatorname{SupF}_{n}(b)=\sup _{t_{1} \leq t \leq t_{2}} F_{t}(b) \Rightarrow_{p} \sup _{\pi_{1} \leq r \leq \pi_{2}} F(r \mid 0) \equiv T(0),
$$

as stated. This means that $G_{n}(\cdot)$ converges uniformly in probability to $G(\cdot)$. By the continuous mapping theorem we conclude

$$
p_{n}=1-G_{n}\left(\operatorname{SupF}_{n}\right) \rightarrow_{d} 1-G(T(\delta))=p(\delta) .
$$

Proof of Theorem 6. Define

$$
S_{t}(b)=\frac{1}{\sigma \sqrt{n}} \sum_{i=1}^{t} x_{n i} u_{i}(b) \tilde{e}_{i} .
$$

The stochastic process $S_{[n r]}(b)$ has an exact distribution as a mean-zero Gaussian process with covariance kernel $\hat{V}_{n}(\min (r, s))$, where

$$
\hat{V}_{n}(r)=\frac{1}{\sigma^{2} n} \sum_{i=1}^{[n r]} x_{n i} x_{n i}^{\prime} \hat{e}_{i}^{2} \Rightarrow V(r) .
$$

Hence $S_{[n r]}^{b} \Rightarrow_{p} N(r)$. The remainder of the proof is identical to Theorem 5 .

\section{References}

[1] Andrews, D.W.K. (1993), "Tests for parameter instability and structural change with unknown change point," Econometrica, 61, 821-856.

[2] Andrews, D.W.K. and W. Ploberger (1994): "Optimal tests when a nuisance parameter is present only under the alternative," Econometrica, 62, 1383-1414.

[3] Basawa, I.V., A.K. Mallik, W.P. McCormick, J.H. Reeves, and R.L. Taylor (1991): "Bootstrapping unstable first-order autoregressive processes," Annals of Statistics, 19, 1098-1101. 
[4] Bose, A. (1988): "Edgeworth correction by bootstrap in autoregressions," Annals of Statistics, 16, 1709-1722.

[5] Carlstein, E. (1986): "The use of subseries methods for estimating the variance of a general statistic from a stationary time series," Annals of Statistics, 14, 1171-1179.

[6] Davidson, J. (1994): Stochastic Limit Theory: An Introduction for Econometricians. Oxford: Oxford University Press.

[7] Efron, B. (1979): "Bootstrap methods: Another look at the jackknife," Annals of Statistics, 7, 1-26.

[8] Engle, R.F. and D.F. Hendry (1993): "Testing superexogeneity and invariance in regression models," Journal of Econometrics, 56, 119-139

[9] Engle, R.F., D.F. Hendry, and J.F. Richard (1983): "Exogeneity," Econometrica 51, 277-304.

[10] Gine, E. and J. Zinn (1990): "Bootstrapping general empirical measures," The Annals of Probability, 18, 851-869.

[11] Hall, P. (1994): "Methodology and theory for the bootstrap," Handbook of Econometrics, Vol. IV, eds. R.F. Engle and D.L. McFadden. New York: Elsevier Science.

[12] Hall, P. and C.C. Heyde (1980): Martingale Limit Theory and Its Application. New York: Academic Press.

[13] Hansen, B.E. (1997): "Approximate asymptotic p-values for structural change tests," Journal of Business and Economic Statistics, 15, 60-67.

[14] Hansen, B.E. (1998): "Sample splitting and threshold estimation," working paper, Boston College.

[15] Hendry, D.F. (1988): "The encompassing implications of feedback versus feedforward mechanisms in econometrics," Oxford Economic Papers, 40, 132-149.

[16] Künsch, H.R. (1989): "The jackknife and the bootstrap for general stationary observations," Annals of Statistics, 17, 1217-1241.

[17] Nyblom, J. (1989): "Testing the Constancy of Parameters Over Time," Journal of the American Statistical Association, 84, 223-230.

[18] Phillips, P.C.B. and M. Loretan (1991): "Estimating long run economic equilibria," Review of Economic Studies, 58, 407-436.

[19] Quandt, R. (1960): "Tests of the hypothesis that a linear regression obeys two separate regimes," Journal of the American Statistical Association, 55, 324-330. 
[20] Saikkonen, P. (1991): "Asymptotically efficient estimation of cointegration regressions," Econometric Theory, 7, 1-21.

[21] Stock, J.H. and M.W. Watson (1993): "A simple estimator of cointegrating vectors in higher order integrated systems," Econometrica, 61, 783-821.

[22] Stock, J.H. and M.W. Watson (1996): "Evidence on structural instability in macroeconomic time series relations," Journal of Business and Economic Statistics, 14, 11-30. 


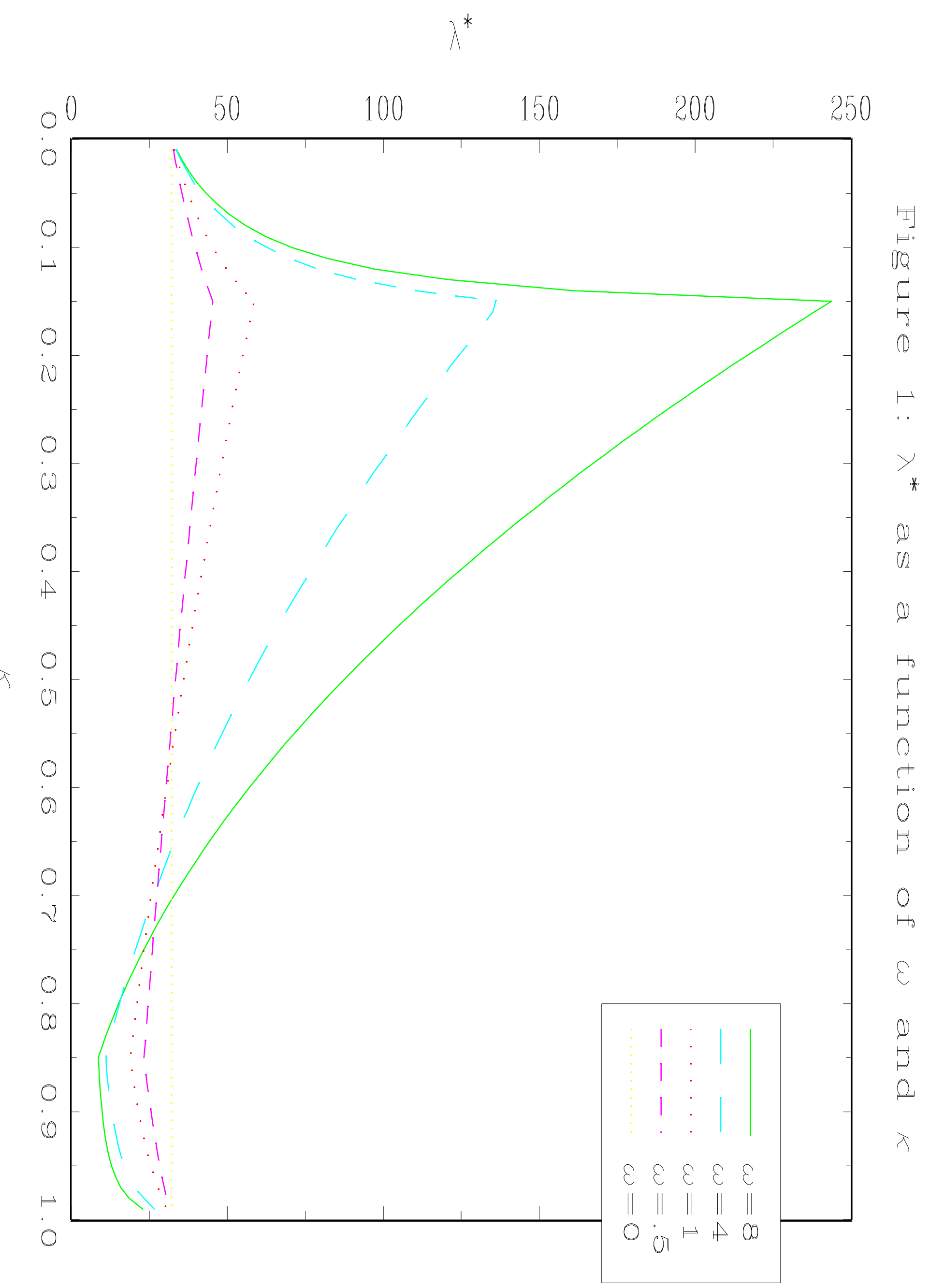


Rejection Frequency

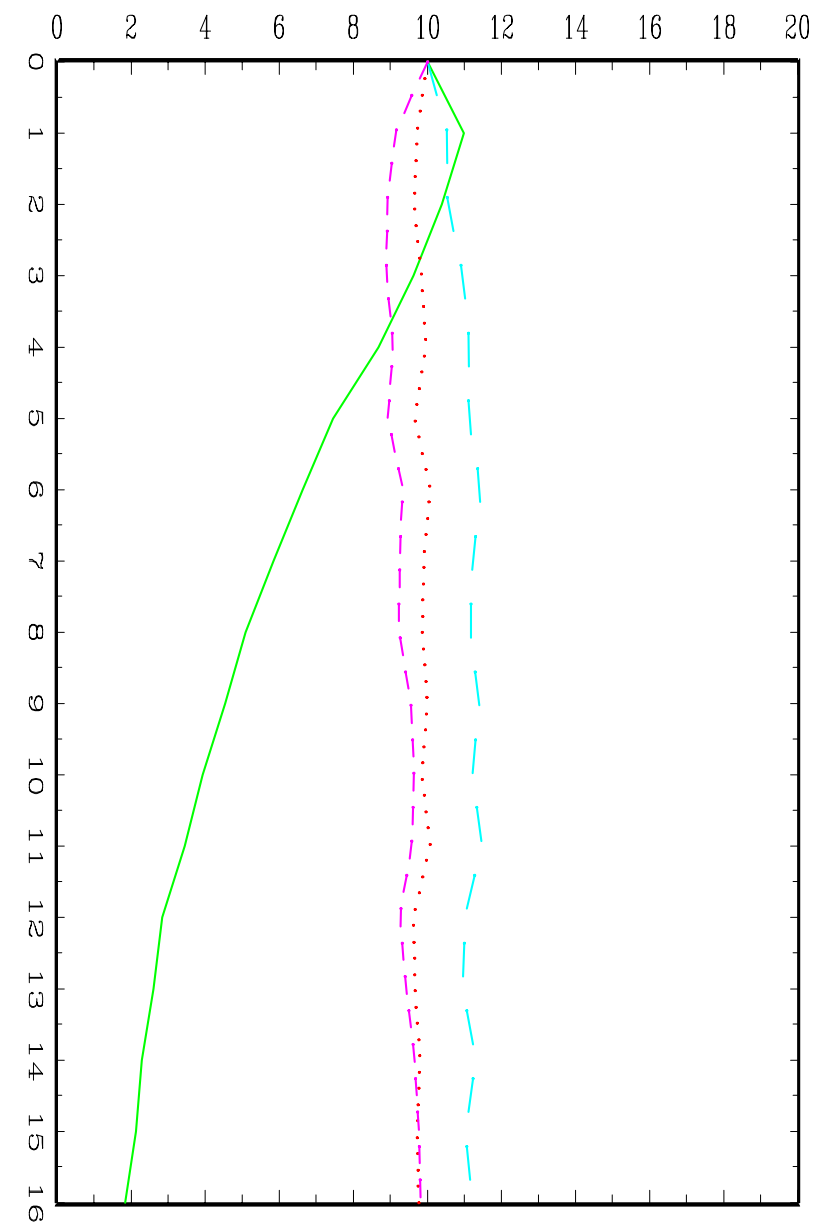

Rejection Frequency

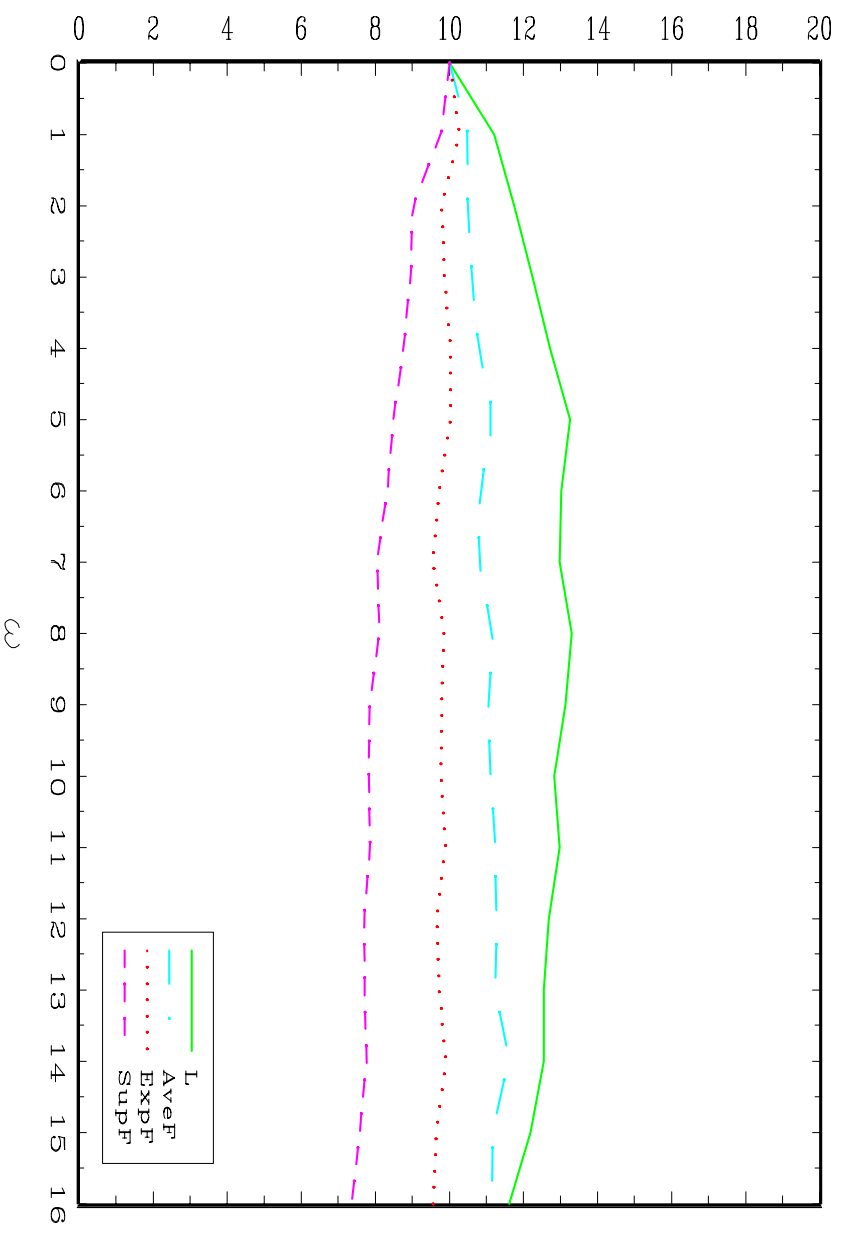

Rejection Frequency

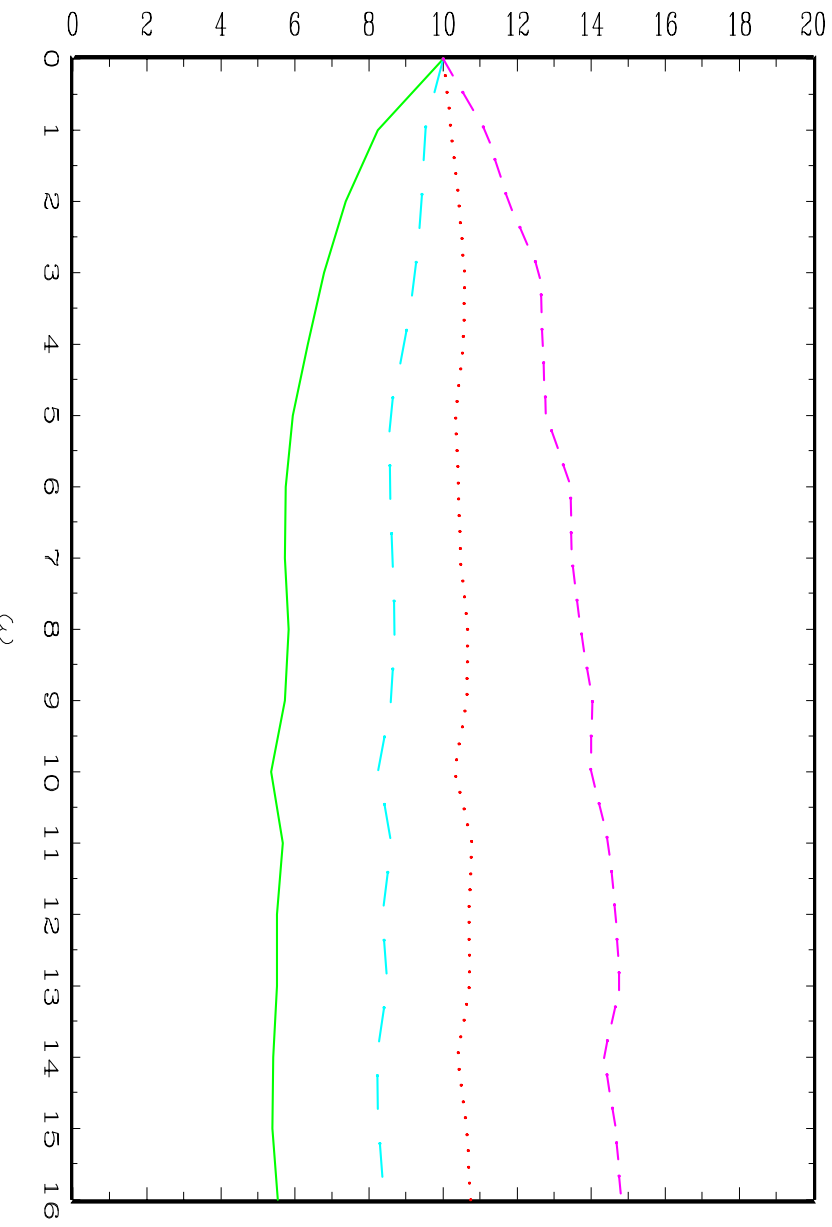

Rejection Frequency

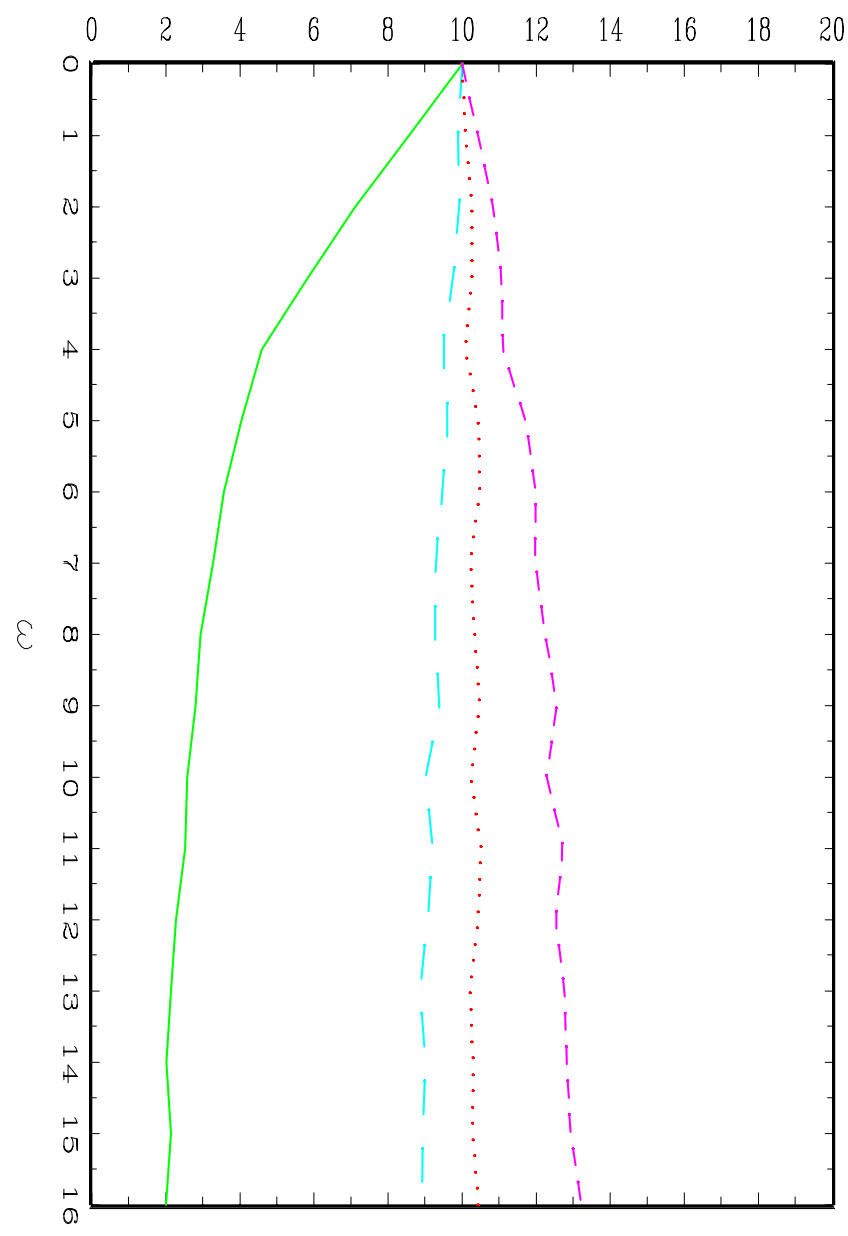

1
50
5
5
5
5
10

N

is $D$ or

4
5
5
5
5
0
0
+5
5
0

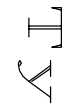

$\nabla$

(1)

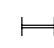

[I]

岁

斿

0

r

$\begin{aligned} & Q \\ &-12\end{aligned}$

or Q

O 
Rejection Frequency

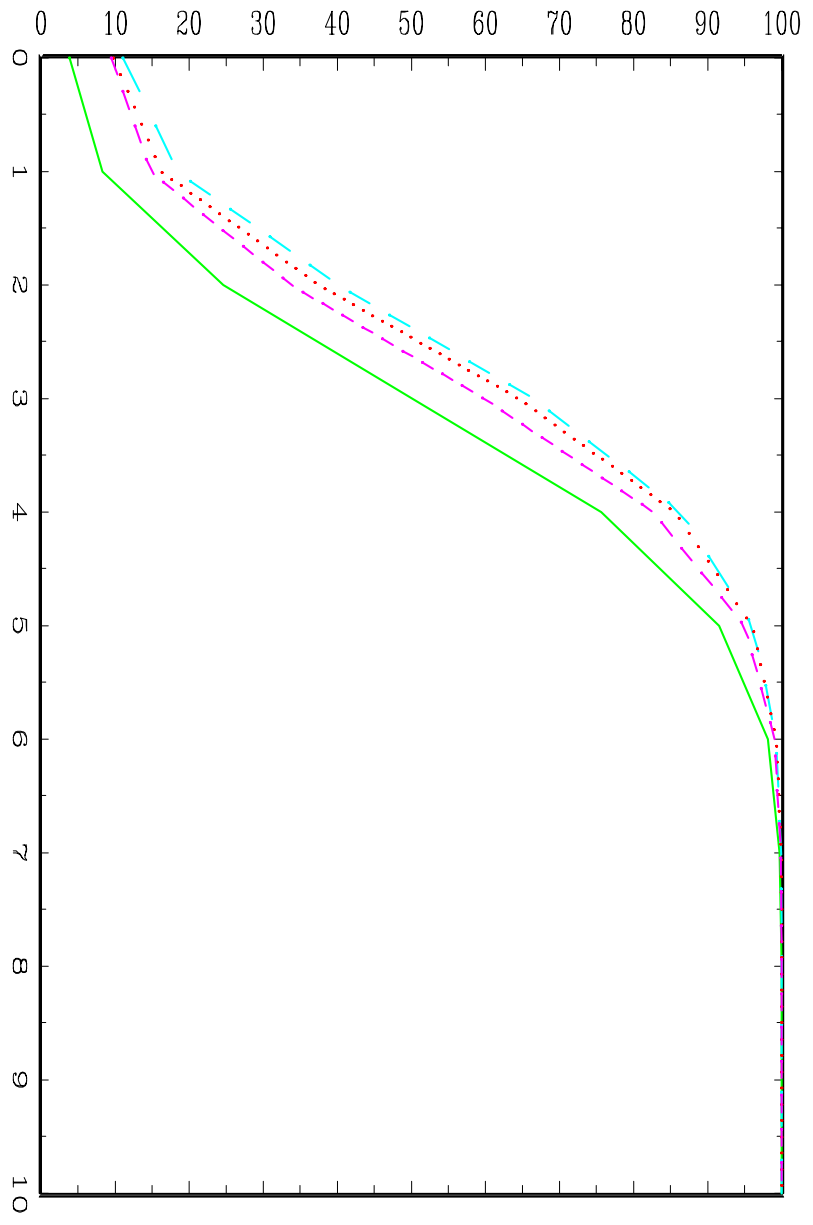

Rejection Frequency

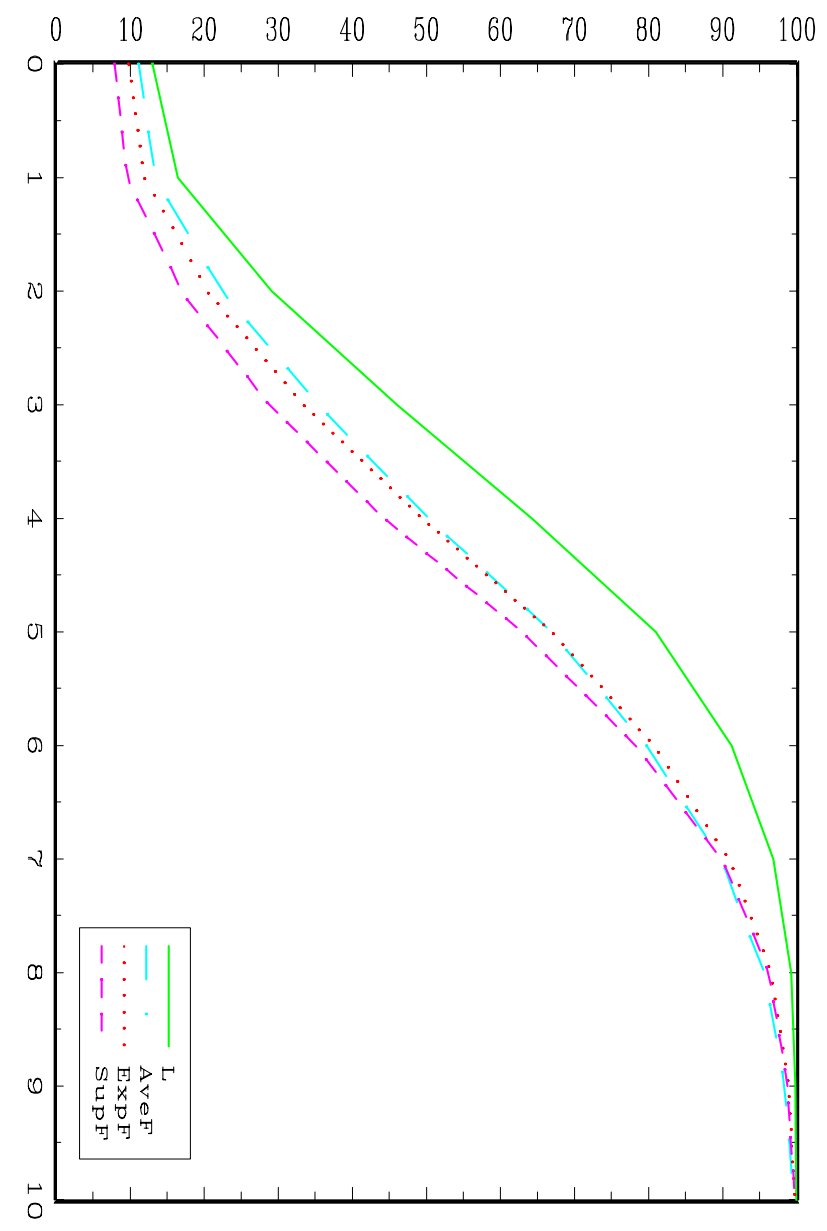

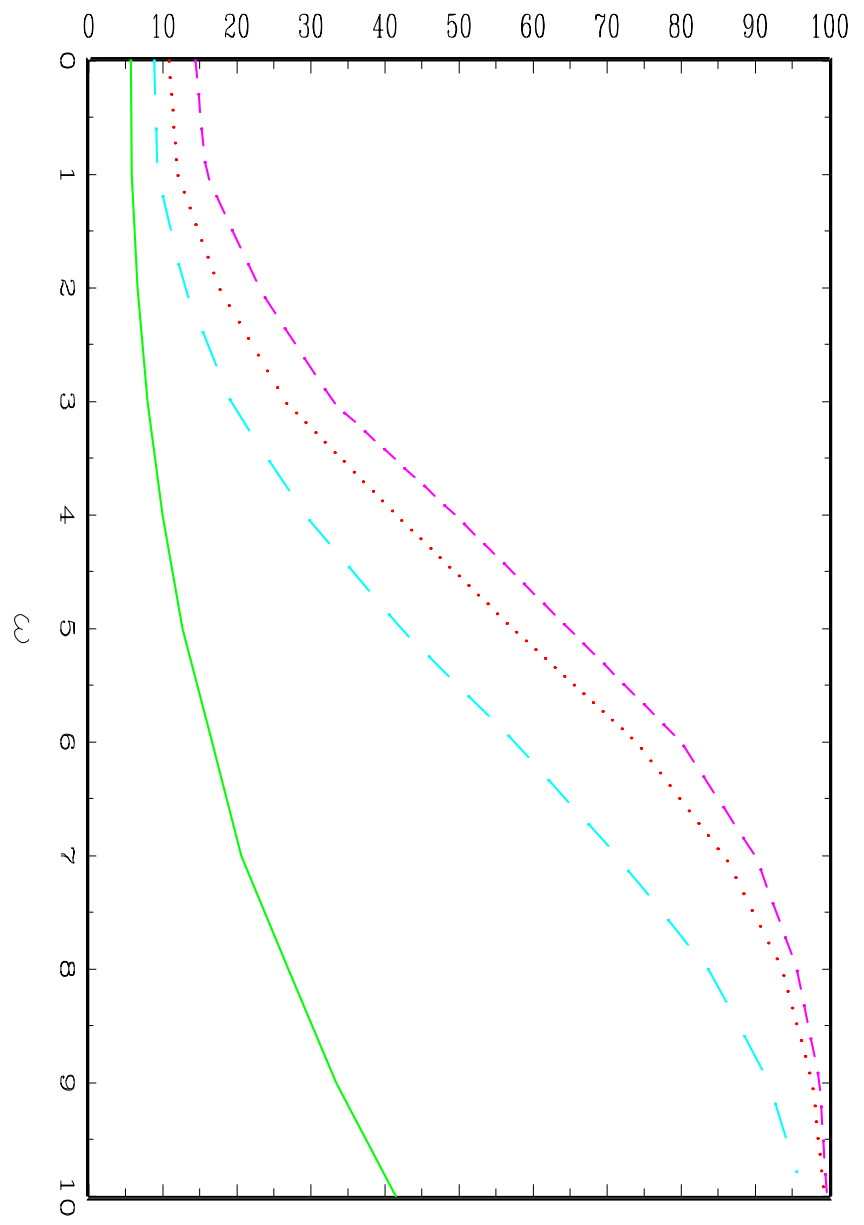

Rejection Frequency

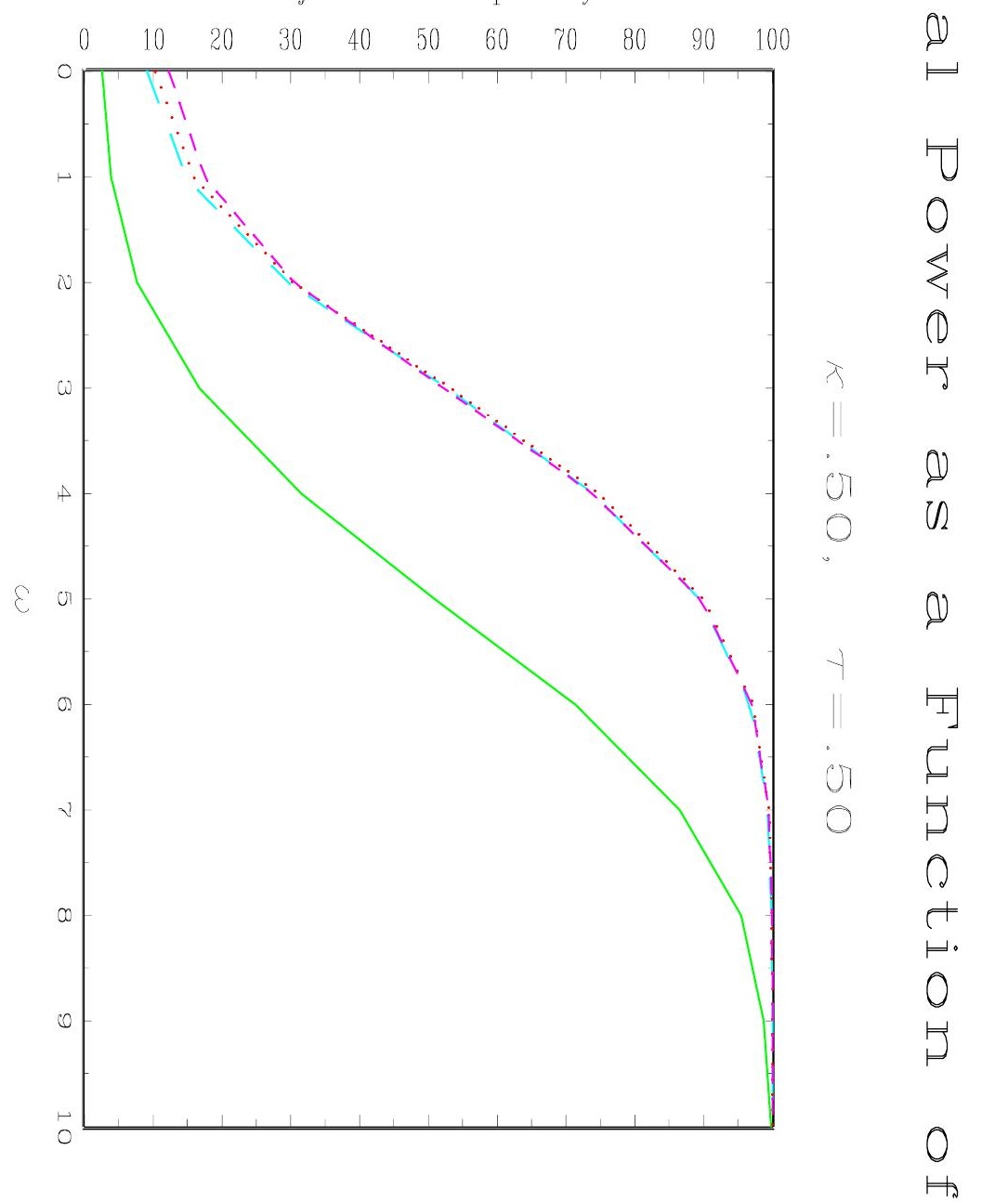

